\title{
Prevalence of Newly Diagnosed End-Stage Renal Disease Patients in a Tertiary Hospital of Central Nepal, Chitwan: A Descriptive Cross- sectional Study
}

\author{
Madhav Ghimire', Shreeju Vaidya', Hari Prasad Upadhyay² \\ 'Department of Nephrology, College of Medical Sciences Teaching Hospital, Bharatpur, Nepal, ${ }^{2}$ Department of \\ Community Medicine, College of Medical Sciences Teaching Hospital, Bharatpur, Nepal.
}

\section{ABSTRACT}

Introduction: End-stage renal disease patients are in rising trend globally, and they have been found to occur predominantly in developing countries. Many studies have been published before, within and across the countries, to know the clinicodemographic profile of end-stage renal disease patients. However, no such studies were done in Chitwan, Nepal. This study's main objective was to find the prevalence of newly diagnosed end-stage renal disease patients.

Methods: A hospital-based descriptive cross-sectional study was carried out in the Department of Nephrology from May 2016 to April 2019. Convenient sampling was done, and all the consecutive new end-stage renal disease patients were included in the study. The ethical approval was taken from the Institutional Review Committee (reference number. 2016/COMSTH/IRC/042). The prevalence and demographic profile of new end-stage renal disease patients were studied. The data were analyzed with appropriate statistical tools.

Results: A total of 250 new end-stage renal disease patients were found among 2200 admitted patients. The prevalence of new end-stage renal disease was found to be $250(11.36 \%)$. Out of 250 patients, males were 156 (62.4\%), and females were 94 (37.6\%). The mean age was $49.6 \pm 15.5$ years. The commonest cause of the incident end-stage renal disease was Type 2 Diabetes mellitus 89 (35.6\%).

Conclusions: The prevalence of new end-stage renal disease was found to be quite high. The commonest cause of the incident end-stage renal disease was Type 2 Diabetes Mellitus.

Keywords: diabetes mellitus; end stage kidney disease; prevalence.

\section{INTRODUCTION}

End-stage renal disease (ESRD) is a major public health problem worldwide and is associated with significant morbidity and mortality. ${ }^{1,2}$ New ESRD patients are rising globally, and the increase has been found to occur predominantly in developing countries. ${ }^{3,4}$

We had realized that the new ESRD patients were increasing in our center. The precise data regarding the true prevalence of ESRD patients lack in Nepal, a retrospective multicentric study done by Hada et al. ${ }^{5}$ revealed that the incidence of ESRD had increased gradually from 3.4 per million populations (pmp) in 1990 to $11.89 \mathrm{pmp}$ in 1999 with an average annual incidence of 6 pmp. However, there were no previous studies regarding the prevalence and clinicodemographic profile of new ESRD patients from Chitwan, Nepal.

Therefore, we thought of doing a study to know the prevalence of new ESRD patients among 2200 admitted renal patients in our center.

\section{METHODS}

A hospital-based descriptive cross-sectional study was carried out in the Department of Nephrology of the college of medical science from May 2016 to April 2019. The ethical approval was taken from the college's

Correspondence: Dr. Madhav Ghimire, Department of Nephrology, College of Medical Sciences Teaching Hospital, Bharatpur, Nepal. Email: madhavghimirenp@yahoo.com, Phone: +977-9855060179. 
Ghimire, et al. Prevalence of Newly Diagnosed End-Stage Renal Disease (ESRD) Patients in a Tertiary Hospital of Central Nepal...

Institutional Review Committee (reference number. 2016/COMSTH/IRC/042), and written informed consents were taken from the patients. Convenient sampling was done, and all the consecutive new ESRD patients, irrespective of their age, sex, and renal diagnosis, were included in the study. A nephrologist made the clinical diagnosis of renal diseases with an experience of $>$ 5 years in clinical Nephrology, and all the diagnoses were supported by relevant biochemistry, radiology, and pathology reports. The standard definitions were used to define the renal diagnosis as per the updated kidney disease improving global outcome (KDIGO) equivalent criteria, wherever applicable.

Sample size for this research was calculated by taking $p$ and $q$ as $50 \%$ with $95 \% \mathrm{Cl}$ and $7 \%$ margin of error. The sample size was calculated using the formula below,

$$
\begin{aligned}
\mathrm{n} & =\mathrm{Z}^{2} \mathrm{pq} / \mathrm{e}^{2} \\
& =(1.96)^{2} \times(0.5) \times(0.5) /(0.07)^{2} \\
& =196
\end{aligned}
$$

By adding a $10 \%$ non-response rate, this research's actual sample size was 216, but this research was conducted among 250 patients.

The patient's demographic profile and cause of new ESRD were noted in the proforma. The data were then entered in the MS XP sheet and then were transferred to the SPSS version 20 (Chicago, IL, USA) program and were analyzed using appropriate statistical tools. The continuous variables were expressed as mean \pm standard deviation (SD) and ratio. The categorical variables were expressed as frequency and percentage.

\section{RESULTS}

Over 3 years, from May 2016 to April 2019, a total of 2200 patients were admitted to the Department of Nephrology. Of them, a total of 250 patients were new ESRD patients. So the prevalence of new ESRD was found to be $11.36 \%$.

Out of 250 new ESRD patients, 156 (62.4\%) were males, and 94 (37.6\%) were females. The mean age of the patient was 49.6 years with a standard deviation of \pm 15.5 years. The minimum age was 17 years and the maximum 85 years. In terms of age distribution, 75

\begin{tabular}{|c|c|c|c|}
\hline \multicolumn{4}{|c|}{$\begin{array}{l}\text { Table 1. Age and gender distribution of new ESRD } \\
\text { patients }(n=250) \text {. }\end{array}$} \\
\hline $\begin{array}{l}\text { Age (in } \\
\text { years) }\end{array}$ & Male n (\%) & Female n (\%) & Total n (\%) \\
\hline $1-20$ & $4(57.1)$ & $3(42.9)$ & $7(2.8)$ \\
\hline $21-40$ & $46(67.7)$ & $22(32.3)$ & $68(27.2)$ \\
\hline
\end{tabular}
$(30.0 \%)$ patients were of age $<40$ years, and $175(70 \%)$ were of age $\geq 40$ years.

\begin{tabular}{|llll|}
$41-60$ & $56(53.9)$ & $48(46.1)$ & $104(41.6)$ \\
$61-80$ & $48(69.6)$ & $21(30.4)$ & $69(27.6)$ \\
$>80$ & $2(100)$ & - & $2(0.8)$ \\
\hline
\end{tabular}

The causes of new ESRD were as shown in table 2. Of the 250 new ESRD patients, kidney biopsies proved diagnosis were in six $(2.4 \%)$ patients. Out of them, $4(1.6 \%)$ were $\lg A$ nephropathy, one $(0.4 \%)$ lupus nephritis (LN), and one $(0.4 \%)$ membranous glomerulopathy (MGN).

\begin{tabular}{|ll|}
\hline \multicolumn{2}{|l|}{ Table 2. Causes of new ESRD patients $(\mathbf{n}=\mathbf{2 5 0})}$. \\
\hline Etiology of new ESRD & $\mathrm{n}(\%)$ \\
Type 2 Diabetes mellitus (T2DM) & $89(35.6 \%)$ \\
Chronic Glomerulonephritis (CGN) & $86(34.4 \%)$ \\
Hypertension (HTN) & $60(24.0 \%)$ \\
Obstructive Uropathy & $6(2.4 \%)$ \\
Autosomal dominant polycystic kidney & \\
disease & $3(1.2 \%)$ \\
Lupus Nephritis & $2(0.8 \%)$ \\
Neurogenic bladder & $1(0.4 \%)$ \\
Congenital anomalies of kidney urinary & $1(0.4 \%)$ \\
tract & $1(0.4 \%)$ \\
Acute Tubular Necrosis (ATN) & $1(0.4 \%)$ \\
Renal Calculus Disease (RCD) & $250(100 \%)$ \\
\hline Total &
\end{tabular}

Out of 250 new ESRD patients, body mass index (BMI) $<18.5 \mathrm{Kg} / \mathrm{m}^{2}$ were seen in $22(8.8 \%)$ patients, between 18.5- $24.9 \mathrm{Kg} / \mathrm{m}^{2}$ were in $143(57.2 \%)$, and $\geq 25 \mathrm{Kg} / \mathrm{m}^{2}$ were in $85(34 \%)$ patients.

\section{DISCUSSION}

The true burden of ESRD faced by Nepal remains unknown due to the limited access of chronic kidney disease (CKD) patients to healthcare facilities, lack of organized CKD management programs, and lack of national registries. Unlike most developed countries, Nepal has yet to develop a national registry of ESRD patients. Available data come from few tertiary referral centers and may therefore not represent the country as a whole. ESRD incidence is rising worldwide and even so in the developing world. ${ }^{1-4}$ To the best of our knowledge, this study was the first of its kind from Chitwan to know the prevalence and clinicodemographic profile of new ESRD patients. A total of 250 new ESRD were identified among 2200 admitted patients for 3 years, this makes the prevalence of new ESRD to be $11.36 \%$, which is quite high for a center like ours, reflecting a high burden of ESRD in this region. Out of 250 new ESRD patients, $62.4 \%$ were males, and $37.6 \%$ were females making a male preponderance with an $M$ : $F$ ratio of $1: 6$, which was concordant with the studies done in India. 6,7 Similar observations of male dominance with an $\mathrm{M}$ : $\mathrm{F}$ ratio of $\sim 2: 1$ were seen in dialysis outcome and practice pattern 
Ghimire, et al. Prevalence of Newly Diagnosed End-Stage Renal Disease (ESRD) Patients in a Tertiary Hospital of Central Nepal...

study (DOPPS) ${ }^{8}$ and Indian study. ${ }^{9,10}$ This dominance of males over the female may reflect the socio-dynamic influence of our society, where a treatment privilege goes to males. It might be because the males are inherently predisposed to develop kidney diseases. This area of research needs multicentric genetic studies with a uniform protocol.

The patient's mean age was 49.6 years with a standard deviation of \pm 15.5 years, which was comparable to a study done by Al-Wakeel et $\mathrm{al}^{11}$ where the mean age of presentation was 53.7 years. However, the mean age was lesser as compared to Okinawa Dialysis Study (OKIDS) Registry (55.9 years), ${ }^{12}$ and united states renal data system (USRDS) 2008 data (64.4 years). ${ }^{13}$ Majority of the patients, $70.0 \%$, were of age $\geq 40$ years, highlighting that ESRD is a middle-age disease (average age of presentation was around 50 years).

The commonest cause of new ESRD in our study was T2DM, 35.6\%, followed by CGN, $34.4 \%$, and HTN $24.0 \%$. There is considerable variation in the causes of ESRD within and across the countries. The etiologic patterns seen in our study were consistent with previous studies. ${ }^{9,14}$ and the CKD registry of India. ${ }^{10}$ However, Sathyan et al. ${ }^{15}$ found CGN 51.0\% and diabetic nephropathy $22.0 \%$ as the most common aetiologies of CKD. Chaudhari et al., ${ }^{16}$ Jha et al. ${ }^{17}$ and Parsi et al. ${ }^{18}$ found diabetic nephropathy, hypertensive nephropathy and chronic glomerulonephritis as the most common etiology of CKD. In a recent populationbased survey, diabetes was the cause of CKD in $41 \%$ of cases. ${ }^{19}$ Similarly in another study ${ }^{20}$ involving several hospitals in India found that $29 \%$ of CKD subjects had diabetic nephropathy. Many of these studies, including our highlight the dominance of diabetic nephropathy as the major cause of ESRD worldwide.
This dominance of diabetes as the major cause may be due to the worldwide rising diabetes mellitus. ${ }^{21,22}$

The majority of the patients, $91.2 \%$ in the new ESRD group ( $n=250$ ), had normal to overweight BMI, making undernutrition a less prevalent problem $8.8 \%$. This might be because most of the patients were above their dry weight, to begin with, due to volume overload, which might have falsely overestimated the BMI and underrepresented the true malnutrition prevalence.

There were few limitations in our study. First, this was an observational study without a control group hence; all the inherent limitations of an observational study were there in the study. This was also a single-center study, so the results might not be generalizable to the whole region and the country, highlighting the need for multicentric studies with a uniform protocol.

\section{CONCLUSIONS}

The prevalence of new ESRD was $11.36 \%$, which is quite high for a center like ours, reflecting a high burden of ESRD in this region, and the commonest cause of incident ESRD was T2DM. These data suggest that ESRD is a growing problem in our region. There is an urgent need to expand the dialysis service centers and kidney transplant programs to accommodate the new and increasing ESRD patients.

\section{ACKNOWLEDGMENTS}

We would like to thank all the patients involved in the study, post-graduate residents, and interns of the nephrology department, who had directly and indirectly helped in the study.

\section{Conflict of Interest: None.}

\section{REFERENCES}

1. Reikes ST. Trends in end-stage renal disease. Epidemiology, morbidity, and mortality. Postgrad Med. 2000;108(1):124-6. [PubMed | Full Text $\mid \underline{\text { DOI] }}$

2. Grassmann A, Gioberge S, Moeller S, Brown G. ESRD patients in 2004: global overview of patient numbers, treatment modalities and associated trends. Nephrol Dial Transplant. 2005;20(12):2587-93. [PubMed | Full Text | DOI]

3. Jha V, Garcia-Garcia G, Iseki K, Li Z, Naicker S, Plattner $\mathrm{B}$, et al. Chronic kidney disease: global dimension and perspectives. Lancet. 2013;382(9888):260-72. [PubMed | Full $\underline{\text { Text }} \mid \underline{\mathrm{DOI}}]$

4. Lozano R, Naghavi M, Foreman K, Lim S, Shibuya K, Aboyans $\mathrm{V}$ et al. Global and regional mortality from 235 causes of death for 20 age groups in 1990 and 2010: a systematic analysis for the Global Burden of Disease Study 2010. Lancet. 2013;380(9859):2095-128. [ㅁubMed | Full Text | DOI]
5. Hada R, Khakurel S, Agrawal RK, Kafle RK, Bajracharya $\mathrm{SB}$, Raut KB. Incidence of end stage renal disease on renal replacement therapy in Nepal. KUMJ. 2009;27:301-5. [PubMed | Full Text | DOI]

6. Dharan KS, John GT, Neelakantan N. Spectrum of severe chronic kidney disease in India: A Clinicopathological study. Natl Med J India. 2006;19:250-2. [ㅁuMed | Full Text]

7. Modi GK, Jha V. The incidence of end-stage renal disease in India: A population based study. Kidney Int. 2006;70(12):2131-3. [PubMed | Full Text | DOI]

8. Hecking M, Bieber BA, Ethier J, Kautzky-Willer A, Sunder-Plassmann G, Säemann MD, et al. Sex-specific differences in hemodialysis prevalence and practices and the male-to-female mortality rate: The dialysis outcomes and practice patterns study (DOPPS) PLoS Med. 2014;11:e1001750. [ubMed | Full Text | DOI] 
Ghimire, et al. Prevalence of Newly Diagnosed End-Stage Renal Disease (ESRD) Patients in a Tertiary Hospital of Central Nepal...

9. Swarnalatha G, Ram R, Prasad N, Dakshinamurty KV. End-stage renal disease patients on hemodialysis: A study from a tertiary care center in a developing country. Hemodial Int. 2011;15:312-9. [PubMed | Full Text | DOI]

10. Rajapurkar MM, John GT, Kirpalani AL, Abraham G, Agarwal SK, Almeida AF et al. What do we know about chronic kidney disease in India: First report of the Indian CKD registry? BMC Nephrol. 2012;13:10. [릴ed | Full Text $\mid \underline{\mathrm{DOI}}]$

11. Al-Wakeel JS, Mitwalli AH, Al Mohaya S, Abu-Aisha H, Tarif N, Malik GH, et al. Morbidity and mortality in ESRD patients on dialysis. Saudi J Kidney Dis Transplant. 2002;13(4):473-7. [․ㅏbMed | Full Text]

12. Iseki $\mathrm{K}$, Tozawa $\mathrm{M}$, Iseki $\mathrm{C}$, Takishita S, Ogawa $\mathrm{Y}$. Demographic trends in the Okinawa Dialysis Study (OKIDS) registry (1971- 2000). Kidney Int. 2002;61:668-675. [PubMed | Full Text | DOI]

13. US Renal Data System. USRDS 2008 Annual Data Report: Atlas of Chronic Kidney Disease and End-Stage Renal Disease in the United States, Bethesda, MD: National Institutes of Health, National Institute of Diabetes and Digestive and Kidney Diseases, 2008.

14. Chandrashekar A, Ramakrishnan S, Rangarajan D. Survival analysis of patients on maintenance hemodialysis. Indian J Nephrol. 2014;24:206-13. [PubMed | Full Text | DOI]

15. Sathyan S, George S, Vijayan P, Jayakumar M. Clinical and epidemiological profile of chronic kidney disease patients in a tertiary care referral centre in South India. International
Journal of Community Medicine and Public Health. 2016 Dec 22;3(12):3487-92. [Full Text | DOI]

16. Chaudhari ST, Sadavarte AV, Chafekar D. Clinical Profile of End Stage Renal Disease in Patients Undergoing Hemodialysis. MVP Journal of Medical Science. 2017 May 22;4(1):8-13. [Full Text]

17. Jha V. Current status of end-stage renal disease care in India and Pakistan. Kidney Int Suppl. 2013;3(2):157-60. [Eull Text I DOI]

18. Parsi MM, Kanni YS, Malhotra V. Etiology and clinico-social profile of chronic kidney disease cases admitted to a dialysis unit in a rural tertiary care hospital. Age;21(40):7. [Full Text]

19. Agarwal SK, Dash SC, Irshad M, Raju S, Singh R, Pandey RM. Prevalence of chronic renal failure in adults in Delhi, India. Nephrol Dial Transplant. 2005;20:1638-42. [PubMed |

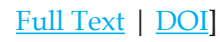

20. Dash SC, Agarwal SK. Incidence of chronic kidney disease in India. Nephrol Dial Transplant. 2006;21:232-33. [PubMed | Full Text | DOI]

21. Peer N, Kengne AP, Motala AA, Mbanya JC. Diabetes in the Africa region: 2013 update for the IDF diabetes Atlas. 2013. [Full Text | DOI]

22. Twagirumukiza M, De Bacquer D, Kips JG, de Backer G, Vander Stichele R, Van Bortel LM. Current and projected prevalence of arterial hypertension in sub-Saharan Africa by sex, age and habitat: an estimate from population studies. J Hypertens. 2011;29(7):1243-52. [PubMed | Full Text | DOI] 